\title{
METHODS OF SOLVING MULTI-AREA ECONOMIC DISPATCH PROBLEMS - A SURVEY AND PROPOSED CANDIDATE SDP APPROACH
}

\author{
Kolapo S. Alli ${ }^{*}$ and Haniph A. Latchman ${ }^{2}$ \\ ${ }^{1,2}$ Faculty of Engineering, The University of the West Indies, Jamaica \\ ${ }^{1}$ Email: kolapo.alli@uwimona.edu.jm *(Corresponding author) \\ ${ }^{2}$ Email: haniph.latman@uwimona.edu.jm
}

\begin{abstract}
Computational intelligence methods may be effectively used to control power system settings automatically to achieve optimal operating power systems objective functions and ensure optimal load flows while fulfilling system constraints. Economic dispatch of power systems involving large interconnected areas or zones require optimum and efficient allocation of the power to ensure efficient transferred output power to the systems in the various zones. One approach in achieving optimum dispatch of the power generated is to model the system as a Multi Area Emission Economic Dispatch (MAEED) optimization problem. One such formulation could be the minimization the running cost and emission objective functions subject to generator power limits, power balance and tie-line capacity constraints. This paper provides a survey of some of the existing research on both single area and multi area economic dispatch problems respectively and discusses the associated methods used in solving these different problems. Based on this study, we propose a candidate approach to address multi area economic dispatch problems using a semidefinite programming (SDP) method and we outline the associated computational and performance advantages.
\end{abstract}

Keywords: MAEED problem, SDP, Tie-line constraint.

https://doi.org/10.47412/DSQG6925

\section{Introduction}

In the optimization of power systems operation, the attainment of the minimum power production cost, efficiency, reliability and better operating condition such as reserve sharing, improved stability and operation under emergencies can be accomplished by interconnecting power systems located in different areas or zones. The topology of the power systems network can either be single area or multi-area, depending on the number of generating units. The resulting Multi-area Economic Emission Dispatch optimization problem involves the optimum placement of the generating units so as to achieve optimal objective functions subject to load balance, generator limit, transmission line and tie-line constraints. The amount of power generated locally in the various zones and transferred to other areas using the solution of MAEED problem is based on the tie-line constraints and the power network constraints. Other advantages of multi-area power systems are to ensure operation continuity, optimum power systems schedule, reduced frequency and power deviation during large interconnected load changes, and transfer of power energy from a particular sub area to other areas. These objectives can be achieved via the tie-lines connections within the zones

The objective of this study is to review some of the existing works on single area and multi area economic dispatch problems, investigate the existing optimization methods and develop an improved method used in solving these economic dispatch problems. Figure 1 shows a typical four-area interconnected power system 
network with tie-lines while Fig. 2 shows the number of publications with the associated algorithms involved. The abbreviations for the algorithms in Fig. 2 are presented below with their definitions obtained from [1].

Where ANN - Artificial Neural Network, BA- Bees Algorithm, BGO- Bi-criterion Global Optimization, CMComponent Model, CT- Computational Time, CUF- Commitment Utilization Factor, DE- Differential Evolution, DP- Dynamic Programming, DS- Direct Search, DWDP- Dantzing Wolfe Decomposition Principle, EM- E-constraint Method, EP- Evolutionary Programming, ES- Expert System, FL- Fuzzy Logic, GA- Genetic Algorithm, GAMS- General Algebraic Modeling System, GSA- Gravitational Search Algorithm, GSM- Grid Service Model, HA- Heuristic Approach, HS- Harmony Search, JM- Jacobin Method, LA- Lagrange“s Algorithm, LI- Lambda Iteration, NFM- Network Flow Modelling, NR- Newton Raphson, PS- Pattern Search, PSO- Particle Swarm Optimization, PTDF- Power Transfer Distribution Factor, QPQuadratic Programming, RMI- Remote Method Invocation, SAMF- Sequential Approach with Matrix Framework, SDP- Semi-definite Programming, TSA- Tabu Search Algorithm, UCA- Unit commitment Approach.

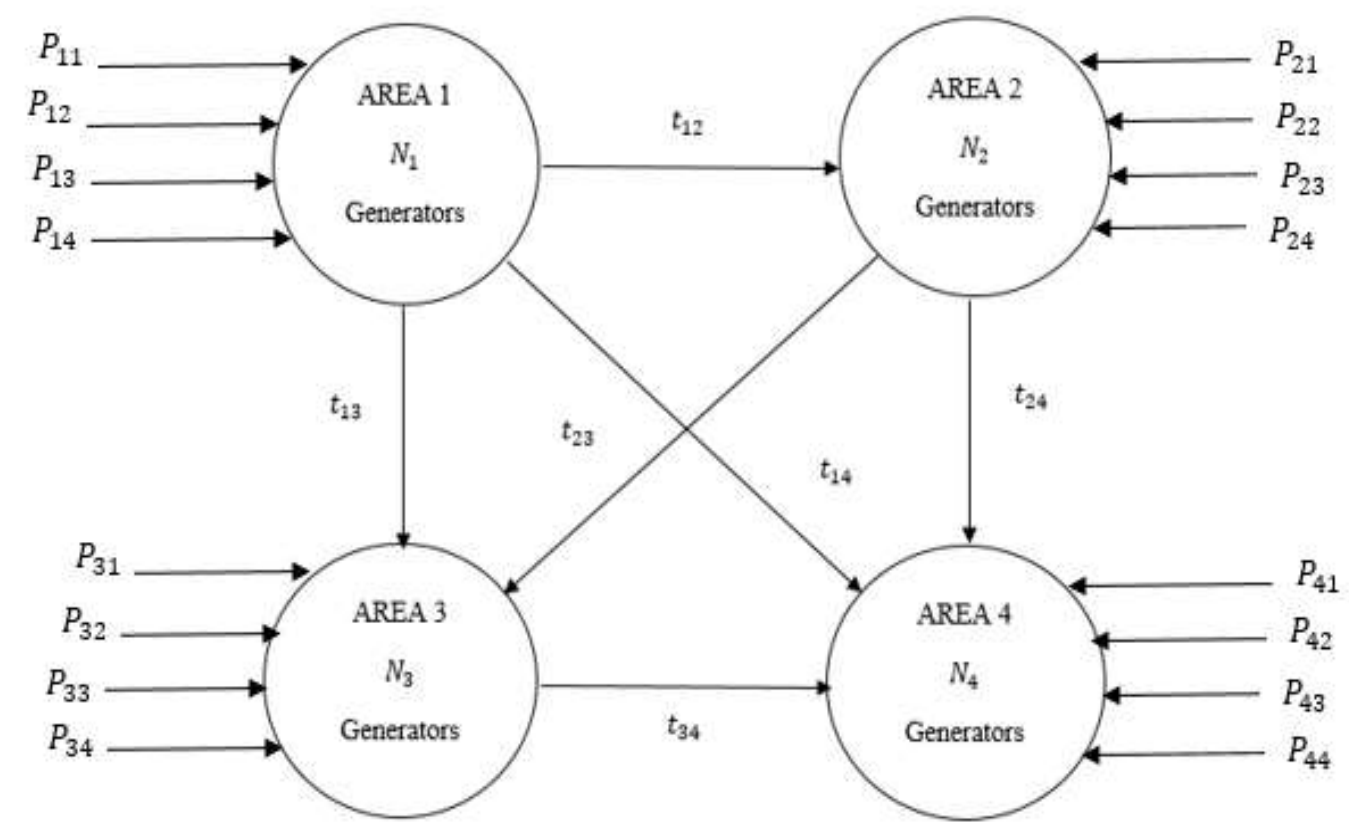

Figure 1: A four Area Network with Tie-lines Power Transfer [1]

Many published works have addressed multi-objective optimization problems in a single area network but only few have considered multi-area multi-objective problems. Some of the optimization techniques that have been used in solving these optimization problems are meta-heuristic methods and have been used to attain only a set of feasible candidate solutions. Examples are Artificial Neural Network (ANN), Virus Optimization Algorithm (VOA), Genetic Algorithm (GA), Particle Swarm Optimization (PSO), Weighted Aggregation, Harmony Search (HS), Differential Evolution (DE), FireFly algorithm (FF), Gravitational Search Algorithm (GSA), and Seeker Optimization Algorithm (SOA). As a result of the stochastic nature of these algorithms, the attainment of the ideal global optimal solutions is not certain as they require multiple runs, generating different solutions in each run. On the other hand, the semidefinite programming (SDP) method is a convex optimization technique, not a population based algorithm and it has been useful in attaining global optimal solution over several runs if the formulation problem is convex and the relaxation is tight. Even for nonconvex problems, semi-definite relaxation of the problem provides a convex estimate that gives an easily computable bound for the problem. SDP has been applied to optimal power flow and economic dispatch problems, and it is currently an active area of research. In the rest of the paper we first present in Section 2, a discussion of the stochastic optimization methods involved in solving 


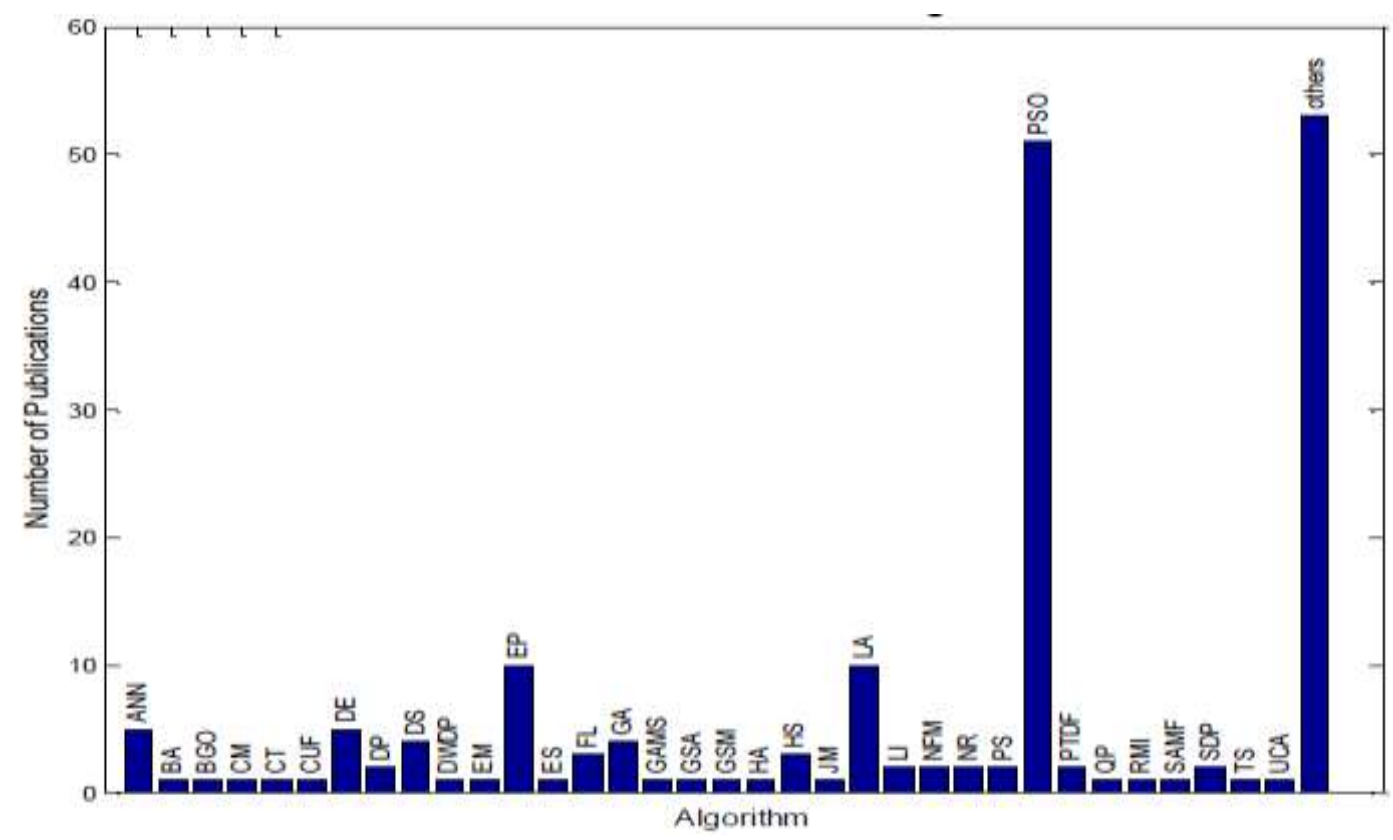

Figure 2: Number of Publications versus Algorithm [1]

multi-objective economic dispatch (MOED) problems on single area networks and multi-area networks respectively. Then in Section 3 we consider the SDP method for solving MOED problems on single area networks as well as multi-area networks.

\section{A Survey of Stochastic Optimization for Single and Multi-Area Networks}

Several studies have considered various problems on multi-objective economic dispatch formulations using the Particle Swarm Optimization (PSO) algorithm. The PSO is a metaheuristic algorithm which mimics the behavior of a flock of birds, and is capable of solving complex problems. The technique operates by using a set of candidates called particles which are accelerated and gradually changed from one generation to another via inter-particle collaboration and contest. A possible solution is then generated by each particle in a swarm. The particles then explore the problem search space finding the best solution. The changes that occur in the movement of every particle are based on its own accumulated knowledge of moving in the environment and that of their neighbors. Some of the applications of PSO related to MOED problems can be found in [1]-[11].

The limitations of the metaheuristic methods are; they involve random iteration for their execution and the attainment of the ideal optimal solutions are not guaranteed in this case. In addition, they are unable to handle problem constraints correctly, which lead to multiple solutions that are non-feasible.

A single area multi-objective economic emission load dispatch was discussed in [12] considering wind power penetration using a gravitational search algorithm approach. This work presented the emission reduction of nitrogen oxides (NOX) and optimized fuel cost, considering both thermal generators and wind power units in an economic emission load dispatch (EELD) problem. The method is an example of the evolutionary technique for which the attainment of the global optimal solutions is not guaranteed.

An effective approach situation using fuzzy self-adaptive learning particle swarm optimization technique for solving dynamic economic emission dispatch problem which integrates wind power units on a single area network has been studied in [13]. The stochastic objective functions are reduced to their equivalent deterministic problems by taking the expectation of the random power variable. The velocity of the random particles is increased by adjusting the inertia weight factor, and/or eliminating local optima, and improving 


\section{The International Conference on Emerging Trends in Engineering and Technology (IConETech-2020) Faculty of Engineering, The UWI, St. Augustine | June $1^{\text {st }}-5^{\text {th }}, 2020$}

the overall performance and efficiency of the particle swarm optimization. The method reported in the work is classified as a heuristic algorithm with inherently multiple optimal solutions over several runs.

In [14], the authors propose a PSO algorithms that incorporates a real-valued mutation (RVM) operator with a view to attain global search capability in solving two real-world economic dispatch (ED) models with nonsmooth cost function considering multiple fuel types and/or valve-point loading effects. The method reported in the work is classified as an evolutionary population-based algorithm and again optimal solutions are not guaranteed.

A single area multi-objective dynamic economic emission dispatch (DEED) problem which incorporates wind power units is presented in [15] and solved by using a PSO algorithm. DEED simultaneously minimizes total electrical energy costs and emissions over a 24-hour time span. The associated stochastic DEED (SDEED) problem is transformed into an equivalent deterministic scenario-based DEED. Thus, the PSO technique is also validated with complicated nonlinear, non-smooth, and non-differentiable SDEED problems, to achieve the best solution for the corresponding scenarios. In this regard, the performance of the method is characterized by various proposed metrics.

In [16], a Quasi-oppositional teaching learning based optimization (QOTLBO) has been proposed as an efficient optimization method used to solve non-linear multi-objective economic emission dispatch (EED) problems of electric power generation with valve point loading implemented on a single area. The proposed method has been performed on 6,10 and 40-units systems to achieve EED solutions. The proposed approach gave improved minimum running fuel cost and emission in a smaller computational time compared to other evolutionary optimization methods. The methods reported in the work are classified as evolutionary algorithms and population based algorithms.

A combined economic and emission dispatch (CEED) model was considered in [17] using a new population based meta-heuristic algorithm known as Virus Optimization Algorithm (VOA) approach for solving this problem. VOA showed better performance results and computational efficiency compared with the other metaheuristic tools (GA, PSO, HS, DE, FF, GSA, and SOA). The methods reported in the work are classified as heuristic, populated based algorithms.

The authors in [18] proposed a novel hybrid particle swarm optimization and gravitational search algorithm (HPSO-GSA), used to solve economic emission load dispatch (EELD) problems, considering various practical constraints such as generator ramp rate limits, the non-convex and discontinuous nature of prohibited operating zones, non-smooth characteristic of valve-point effects, multiple fuels type of generation units, and transmission losses in feasible power systems. The methods reported in the work are classified as evolutionary algorithms and it is limited to a single area network.

An optimization problem has been solved in [19] using the - Gbest-guided Artificial Bee Colony optimization algorithm (GABC) for analyzing the economic benefits in terms of fuel cost of power systems. The power formulation included both the expected penalty cost and expected reserve cost to model wind power generation instability applied on a single area network. The technique used in the work is a population-based algorithm.

A Robust Economic/Emission Dispatch (REED) model integrating wind power was presented in [20] and solved by a Latin hypercube sampling (LHS) technique to achieve minimum running cost and emission reduction applied on a single area network. Analysis results showed total optimal solutions for objective functions is achieved in the presence of wind power uncertainties and flexible operation of a carbon capture and storage (CCS) which has advantages over various carbon reduction indices. The technique used in the work is population based algorithm, therefore, ideal optimal solutions are not guaranteed, due to their random nature.

A new Multi-Objective Evolutionary Algorithm based on Decomposition (MOEA/D) optimization technique has been developed in [21] to solve a single area economic dispatch problem in optimizing the cost and emission of thermal power systems integrated with wind power units. The Weibull probability distribution 


\section{The International Conference on Emerging Trends in Engineering and Technology (IConETech-2020) Faculty of Engineering, The UWI, St. Augustine | June $1^{\text {st }}-5^{\text {th }}, 2020$}

function is used to characterize wind power instability and system constraints with random variables are considered in the study. The analysis results reported in the work show that MOEA/D technique generated better minimum solutions in less computational time compared to other similar optimization methods. The technique used in the work is a population-based algorithm.

The development of an optimal wind-thermal dispatch in isolated single area power systems solved by an iterative direct search method (DSM) was introduced by [22]. Simulations conducted gave information about the level of understanding of the wind generator capacity as related to production cost and planning issues. The technique used in the work is population-based algorithm and was mainly used on a single area network.

The authors in [23] have solved dynamic economic emission dispatch (DEED) power system problems, which involve a constraint handling scheme introduced to deal with complex constraints using a Group Search Optimizer with Multiple Producers (GSOMP) evolutionary algorithm. The DEED is solved in time intervals which are structured into 24 divisions. Simulations were conducted on IEEE 30-bus and 118-bus systems respectively, to investigate the efficiency of GSOMP for solving DEEDs over those of multi-objective particle swarm optimizer (MOPSO) and non-dominated sorting genetic algorithm-II (NSGA-II). The technique used in the work is population based algorithm.

A Gbest guided artificial bee colony algorithm for solving a single area environmental/economic dispatch considering wind power units has been presented by [24]. In this study, a Weibull probability distribution function is established in characterizing the random nature of the wind power. Both overestimation and underestimation costs of available wind power are calculated the optimization process. Three standard test systems which include different technical constraints such as valve loading effect, prohibited zones and ramp rate limits are considered to determine the effectiveness of the optimization method. The optimal results obtained using the proposed method are compared to other related optimization techniques and achieved better solution accuracy and convergence results. The technique used in the work is a population based algorithm.

A new Strength Pareto Evolutionary Algorithm (SPEA) based approach has been proposed in [25] for solving Environmental/Economic power Dispatch (EED) as a multi-objective optimization problem with competing and non-proportionate objectives. A diversity-preserving mechanism to overcome premature convergence and search bias problems has been employed by the proposed approach. A hierarchical clustering algorithm is required to give the decision maker a representative and manageable Pareto-optimal set. Furthermore, this work employed the use of fuzzy set theory to extract the best compromise non-dominated solution. The proposed approach has been carried out on a standard test system to perform several optimization runs. The results reported show that the proposed technique demonstrated the ability to generate well-distributed Paretooptimal solutions of the multi-objective EED problem in a single run. The technique used in the work is a population based algorithm.

A two-stage Stochastic Optimization has been proposed in [26] for solving Optimal Power Flow under Renewable Generation Uncertainty. For the two-stage stochastic classical economic dispatch problem with AC power flow constraints, a non-convex optimization formulation that is central to power transmission and distribution through an electricity grid was studied. The analysis show that the proposed method described was efficient and usable even when solving large practical problems.

A multi area emission economic dispatch (MAEED) problem has been investigated and solved with a PSO technique in [27]. In this work, two objective functions which are fuel cost and emission objective functions are optimized simultaneously subject to system constraints such as generators power limits, power balance, tie-line and reserve power constraints. Also, a set of Pareto solutions from the MAEED problem is developed by PSO method. This study was tested on a 4 area 6-unit network to determine the efficiency of the proposed method and optimization results generated from PSO method are compared with other existing methods.

A two area power systems economic dispatch problem subject to transmission capacity constraints was solved in [28] using a direct search method. This method has been used to solve different polynomial cost functions 
subject to equality and inequality constraints. This work investigated the impact of tie-line capacity between the two areas on the total fuel cost for running the power systems.

An algorithm called Ant Lion optimizer was considered in [29] for solving multi area multiple objective dispatch problems considering multiple fuel generating units, subject to multiple constraints. The best compromise solution is obtained from multi-objective problems by adopting a fuzzy decision mechanism in the algorithm. The efficiency of the method was validated on a standard 10 generating unit- network with a range of operational scenarios. Simulation analysis results show that the proposed method performed well in terms of quality compared to results obtained from the other techniques.

A Real-Coded Genetic Algorithm has been proposed in [30] to solve multi area economic dispatch problem with tie-line constraint and other system constraints. The optimization results obtained from the proposed method are compared with particle swarm optimization using time-varying acceleration coefficients (PSOTVAC) to evaluate the performance of the proposed method.

A multi area economic dispatch problem was proposed and solved in [31] using a Hybrid Cuckoo Searching Algorithm. This hybrid Cuckoo method involves the combination of Cuckoo searching algorithm and teaching learning based optimization method, to achieve better accuracy. The optimization results obtained from the hybrid Cuckoo method are compared with Cuckoo method and Teaching- learning based algorithm to examine its efficiency.

Other area of applications of evolutionary algorithms in solving both single area and multi-area economic dispatch problems can be found in [32]-[37].

\section{The Existing Works on SDP Methods for Solving Single Area and Multi Area MOED Problems.}

As mentioned earlier, the semi-definite programming (SDP) method is a convex technique, not a populationbased algorithm and it has been useful in attaining global optimal solution over several runs if the formulation is convex and the relaxation is tight. Even for non-convex problems, semi-definite relaxation of the problem provides a convex estimate that gives an easily computable bound for the problem.

A Semi-definite Programming (SDP) Weighted Sum Based Technique for Solving Stochastic Multi-Objective Economic Dispatch Problems Incorporating Combined Heat and Power (CHP) Units was presented in [38]. Simulations were conducted on a modified IEEE 6-unit and 20-unit single area network. The SDP technique achieved minimum cost objective function compared to other evolutionary methods reported in the literature. Results showed that the semi-definite programming based weighted sum method has inherently good convergence properties and can have its diversity property improved through weight adaptation.

A semidefinite approach for solving stochastic multi-objective economic dispatch problem incorporating both CHP units and wind power units was proposed in [39]. The stochastic multi-objective model was first converted into its deterministic equivalent by taking the mean of all the involved random values assuming they are normally distributed. The multi-objective problem was recast in matrix form as a SDP relaxation problem and subsequently solved with a MATLAB programming suite. The system inequality and equality constraints uncertainty were entered into YALMIP, a linear matrix inequality parser. Simulations were performed on modified IEEE 6, 15 and 20 unit- networks with 2 CHP units and 20 wind parks for each of the networks. A comparative study was also conducted to demonstrate the effectiveness of the proposed method whereby the results of problem reformulations including stochastic and deterministic models of power dispatch were investigated and then compared with the results of the existing techniques reported. However, in the generation of the Pareto-front solutions, ideal minimum points were used in the determination of the maximum spread out of the Pareto solutions by the algorithm. This involves the use of a standard weighted sum method in generating the Pareto-optimal solution between two objective functions. The optimization results are within the order of magnitude $3.5 \%$ reductions in the case of the modified IEEE 6-unit system. 
Additionally, the SDP method achieved the lowest values of the optimized objective functions and the Pareto set is formed faster in a single run compared to modified particle swarm optimization (MOPSO), genetic algorithm (GA) and weighted aggregated (WA) methods.

High order polynomial functions expressing the fuel cost and emission objective economic dispatch models in a single area network have been solved using semidefinite programming technique, as reported in [40]. In this work, the polynomial functions of both objective functions are approximated to a single objective function using a weighted sum method. The standard polynomial functions are converted into SDP formats in a matrix representation. Different degrees of approximations of problems in SDP format are solved with a semidefinite programming technique. Simulations were conducted on IEEE 6- and 13- unit systems respectively, to evaluate the effectiveness of the proposed methods, compared to other evolutionary methods reported in the literature. The optimization results obtained from SDP technique show improved convergence properties and better exploration of solution points on the Pareto front compared to other methods.

In the paper, "Semi-Definite Programming in solving Power Output Control problem in a Wind Energy Conversion System" [41], the stochastic nature of wind speed fluctuation on control inputs and system outputs has been addressed using random constraints instead of deterministic constraints forms in the prior literature. In addition, two different models are considered namely, Gaussian distribution for the norm bounded wind speed measurement error, and the formulation of the problem as a min-max optimization problem. The models are then reformulated as semi-definite programming optimization problems that can be solved effectively with existing software tools. Conclusively, the simulation results presented show the efficiency of the proposed technique. The results presented in this work were on a dynamic wind energy conversion system. Other application of SDP on MEED problems are presented in [42].

\section{Proposed Candidate SDP Approach for Solving Multi-Area Economic Dispatch Problems}

Semi-definite programming (SDP) is considered to be an extension of linear programming. SDP is a convex optimization technique that minimizes objective function(s) subject to the constraint that an affine combination of symmetric matrices is positive semidefinite [38]. In addition, the componentwise inequalities between vectors are substituted by matrix inequalities, or similarly, the first orthant is replaced by the cone of positive semidefinite matrices

SDP relaxations for minimizing a nonconvex quadratic function are given as follows [38]:

$$
\begin{array}{cc}
\min \left\{x^{T} \gamma_{i} x+\beta_{i}{ }^{T} x+\alpha_{i}\right\} & \\
\text { subject to } \quad P_{\text {min }} & \leq \bar{P}_{\imath} \leq P_{\max } \\
X & =x x^{T}
\end{array} \quad i=1,2, \ldots N
$$

where $x \in \mathbb{R}^{n}, \gamma_{i} \in \mathbb{R}^{n x n}, \beta_{i} \in \mathbb{R}^{n}, \alpha_{i} \in \mathbb{R}^{n}$ and $\bar{P}_{l} \in \mathbb{R}^{n}$. The Eq. (1) is non-convex optimization problem, which is difficult to solve if the problem is not a positive semidefinite. It is best to relax Eq. (1) to a convex optimization problem so as to achieve a global optimal solution for the problem in (1), which generates a tight lower bound on the optimal value with reduced computational time. Also, the equality constraint in Eq. (1) is replaced by a semidefinite inequality $X \geq x x^{T}$ which should be included to obtain an SDP relaxation. However, (1) becomes;

$$
\begin{array}{cl}
\min \left\{\operatorname{trace}\left\langle\gamma_{i} X\right\rangle+\beta_{i}{ }^{T} x+\alpha_{i}\right\} & \\
\text { subject to } & P_{\min } \leq \bar{P}_{l} \leq P_{\max } \\
& \left(\begin{array}{cc}
1 & x^{T} \\
x & X
\end{array}\right) \geq 0
\end{array}
$$


Finally, the quadratic expression in Eq. (2) can be expressed in internal SDP matrix form as follows [38]:

$$
\begin{aligned}
\min \left\langle\left[\begin{array}{ll}
\gamma_{i} & \beta_{i} \\
\beta_{i}{ }^{T} & \alpha_{i}
\end{array}\right]\left[\begin{array}{cc}
1 & x^{T} \\
x & X
\end{array}\right]\right\rangle & \\
\text { subject to } & P_{\min } \leq \bar{P}_{l} \leq P_{\max } \\
& \left(\begin{array}{cc}
1 & x^{T} \\
x & X
\end{array}\right) \geq 0
\end{aligned}
$$

A particular problem with existing evolutionary algorithms, is their inability to generate a single ideal optimum solution as they require multiple run and different solutions are obtained in each run. Therefore, this leads to keeping of statistical data for finding the best and worst optimal solutions.

As shown in the above survey, the SDP approach has produced reliable results for single area economic dispatch problems, associated with the convex non-iterative optimization.

We therefore propose to use a linear bi-objective multi-area economic dispatch problem, reformulated in SDP format by converting the problem into its matrix representation as a SDP relaxation problem using a MATLAB suite, with the system equality and inequality constraints entered into a SElf-DUal MInimization (SEDUMI) parser. Two main cases will be considered.

1. Simulation analysis will be conducted on an IEEE 16-unit system interconnecting four zones to form a 4 area network, as well as on an IEEE 4 areas 40-unit network, with 10-units in each area, to investigate the performance of the SDP method in terms of accuracy and computational efficiency.

2. The results of problem formulations for different cases of both tie-line and without tie-line capacities models respectively on power dispatch using SDP technique will be investigated and also, the optimal solutions for different objective functions obtained from the proposed SDP technique will be compared with the Multi-objective Particle Swarm Optimization (MOPSO), Multi-objective Evolutionary Algorithm (MOEA) and Weighted Aggregation (WA).

The SDP approach is expected to yield minimum fuel cost and reduced emission objective functions for different cases of both tie-line and without tie-line transfer capacities respectively when compared with the optimization results from other methods reported in the literature.

In conclusion, we expect that the SDP technique will be able to give an overall reduction in the fuel cost of the power systems obtained when the tie-line power sharing between areas is considered in the optimization model compared to the case when the tie-line transferred power is not included in the optimization formulations. Finally, the SDP approach will be able to achieve better accuracy and generate the lowest cost compared to other methods.

Future work will present the importance of transfer tie-line capacity constraint when considered in the optimization model and its contribution to optimum production of power generation, total running cost and emission reductions using semidefinite programming. A block diagram of the proposed SDP approach is shown in Fig. 1.

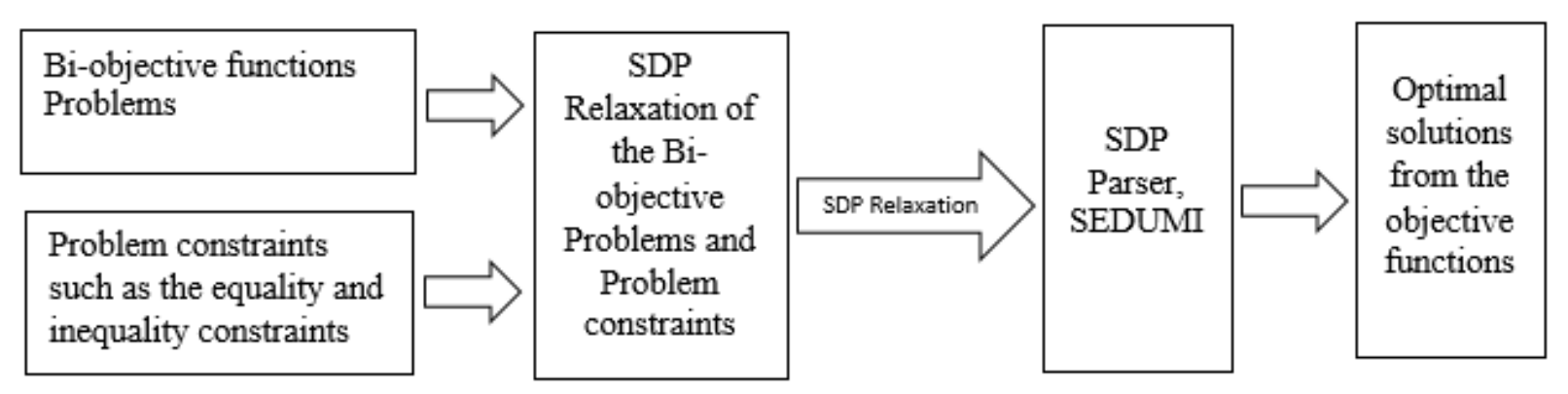


Figure 3. The block diagram for the MAEED Problem solutions using the SDP approach

\section{Conclusion}

This survey has discussed a number of studies that have addressed both single area and multi area economic dispatch problems and some of the existing optimization methods involved in solving these problems. It also addressed the way forward in handling multi area economic dispatch problems and has the potential to generate better solutions and can achieve global optimal solutions, improve performance and convergence speed. These solutions will assist the power systems administrator to achieve an improved decision that will lead to a more effective dispatch of power.

\section{References}

[1]. S. Krishnamurthy (2013). Ph.d Thesis “ Development of Decomposition Methods for Solution of a Multiarea Power Dispatch Optimisation Problem" Cape Peninsula, University of Technology.

[2]. P. Ajay, D. Raj, T. Palanivelu and R. Gnanadass, (2007). Optimal Power Flow Solution for Combined Economic Emission Dispatch Problem using Particle Swarm Optimization Technique, Journal of Electrical Systems 3(1): 13-25.

[3]. E. Azadani, S. Hosseinian, B. Moradzadel, B. and P. Hasanpor, (2008). Economic Dispatch in Multi-area using Particle Swarm Optimization in Electricity Market, Power System Conference, 12th International Middle-East pp. 559-564.

[4]. J. Hetzer, C. David, C. Yu, and K. Bhattarai, (2008). An Economic Dispatch Model Incorporating Wind Power, IEEE Transactions on Energy Conversion 23(2): 603-611.

[5]. G. Piperagkas, A. Anastasiadis, and N. Hatziargyriou, (2011). Stochastic PSO-based and Power Dispatch under Environmental Contraints Incorporating CHP and Wind Power Units, Electric Power Systems Research. 81(1): 209-218.

[6]. L. Wang and C. Singh, (2006b). Tradeoff between Risk and Cost in Economic Dispatch. Including Wind Power Penetration Using Particle Swarm Optimization, International Conference on Power System Technology pp. 1-7.

[7]. C. Carlos, T. Gregorio and S. Maximino, (2004). Handling Multiple Objectives with Particle Swarm Optimization, IEEE Transactions on Evolutionary Computation 8(3): 345-356.

[8]. P. Jong-Bae, L., Ki-Song, S. Joong-Rin, and Y. Kwang, (2005). A Particle Swarm Optimization for Economic Dispatch with Nonsmooth Cost Functions, IEEE Transactions on Power Systems 20(1): 275-282.

[9]. W. Lingfeng and S. Chanan, (2008). Balancing Risk and Cost in Fuzzy Economic Dispatch Including Wind Power Penetration Based on Particle Swarm Optimization, Electrical Power and Systems Research 78: 1361-1368.

[10]. A. Shubham, B. Panigrahi and M. Tiwari, (2008). Multi-objective Particle Swarm Algorithm with Fuzzy Clustering for Electrical Power Dispatch, IEEE Transactions on Evolutionary Computation 12(5): 567-578.

[11]. A. Shubham and B. K. Panigrahi, (2005). A New Particle Swarm Optimization Solution to Nonconvex Economic Dispatch Problems, IEEE Transactions on Power Systems 22(1): 380-389.

[12]. M. Soumitra, B. Aniruddha and H. Sunita, (2013). Multi-objective Economic Emission Load Dispatch Solution using Gravitational Search Algorithm and Considering Wind Power Penetration, Electrical Power and Energy Systems 44: 282-292.

[13]. B. Firouzi, E. Farjah, and R. Abarghooee, (2013). An Efficient Scenario-Based and Fuzzy Self-Adaptive Learning Particle Swarm Optimization Approach for Dynamic Economic Emission Dispatch Considering Load and Wind Power Uncertainties, Journal of Energy, Energy 50: 232-244.

[14]. H. Lu, P. Sriyanyong, Y. Song and T. Dillon, (2010). Experimental Study of a New Hybrid PSO with Mutation for Economic Dispatch with Non-Smooth Cost Function, Electrical Power and Energy Systems 32: 921-935.

[15]. J. Aghaei, T. Niknam, R. Abarghooee and J. Arroy, (2013). Scenario-Based Dynamic Economic Emission Dispatch Considering Load and Wind Power Uncertainties, Electrical Power and Energy Systems 47: 351-367. 
[16]. P. Roy and S. Bhui, (2013). Multi-objective Quasi-Oppositional Teaching Learning Technique for Economic Emission Load Dispatch Problem, Electrical Power \& Energy Syst 53: 937-948.

[17]. Y. Liang and R. Juarez, (2014). A Normalization Method for Solving the Combined Economic and Emission Dispatch Problem with Meta-heuristic Algorithms, Electrical Power \& Energy Syst 54: 163-186.

[18]. S. Jiang, Z. Ji and Y. Shen, (2014). A Novel Hybrid Particle Swarm Optimization and Gravitational Search Algorithm for Solving Economic Emission Load Dispatch Problems with Various Practical Constraints, Electrical Power and Energy Systems 55: 628-644.

[19]. R. Roy and H. Jadhav, (2015). Optimal Power Flow Solution of Power System Incorporating Stochastic Wind Power Using Gbest Guided Artificial Bee Colony Algorithm, Electrical Power and Energy Systems 64: 562-578.

[20]. Z. Lu, S. He, T. Feng, X. Li, X. Guo and X. Sun, (2014). Robust Economic/Emission Dispatch Considering Wind Power Uncertainties and Flexible Operation of Carbon Capture and Storage, Electrical Power and Energy Systems 63: 285-292.

[21]. Y. Zhu, J. Wanga and B. Qu, (2014). Multi-objective Economic Emission Dispatch Considering Wind Power Using Evolutionary Algorithm Based on Decomposition, Electrical Power and Energy Systems 63: 434-445.

[22]. C. Chen, T. Lee and R. Jan, (2006). Optimal Wind-thermal Coordination Dispatch in Isolated Power Systems with Large Integration of Wind Capacity, Energy Conversion and Management 47: 3456-3472.

[23]. C. Guoa, J. Zhana and Q. Wub, (2012). Dynamic Economic Emission Dispatch Based on Group Search Optimizer with Multiple Producers, Electric Power Systems Research 86: 8-16.

[24]. H. Jadhav and R. Roy, (2013). Gbest Guided Artificial Bee Colony Algorithm for Environmental/ Economic Dispatch Considering Wind Power, Expert Systems with Applications 40: 6385-6399.

[25]. M. Abido, (2003). Environmental/Economic Power Dispatch Using Multi-objective Evolutionary Algorithms, IEEE Transactions on Power Systems 18(4): 785-792.

[26]. P. Dzung and G. Soumyadip, (2012). Two-stage Stochastic Optimization for Optimal Flow under Renewable Generation Uncertainty, ACM Transaction on Modeling and Computer Simulation 5(2): 288295. [27]. L. Wang and C. Singh, "Reserve-constrained Multi-area Environmental/ Economic Dispatch Based on Particle Swarm Optimization with Local Search," Engineering Applications of Artificial Intelligence, vol. 22, no. 2009, pp. 298-307, October 2008.

[28]. M. Zarei, A. Roozegar, R. Kazemzadeh, and J.M. Kauffmann (2007). "Two Area Power Systems Economic Dispatch Problem Solving Considering Transmission Capacity Constraints" World Academy of Science, Engineering and Technology International Journal of Electrical, Computer, Electronics and Communication Engineering Vol:1, No:9, 2007.

[29]. P. Balachandar, S. Ganesan, N. Jayakumar and S. Subramanian, (2017) "Economic/ Environmental Dispatch of an Interconnected Power System considering Multiple Fuel Sources," International Conference on circuits Power and Computing Technologies, ICCPCT, 2017.

[30]. H. T. Binh and T. K. Toan, (2013) "Real-Coded Genetic Algorithm for Solving Multi-Area Economic Dispatch Problem," IEEE Symposium on Computational Intelligence for Engineering Solutions, CIES, pp. 97101, 2013.

[31]. K. P. Nguyen, N. D. Dinah and G. Fujita, "Multi-area Economic Dispatch Using Hybrid," IEEE International Universities Power Engineering Conference, UPEC, September 2015.

[32]. Y. Guo, L. Tong, W. Wu and B. Zhang, "Coordinated Multi-Area Economic Dispatch via Critical Region Projection," IEEE Transaction on Power Systems, vol. 32, no. 5, pp. 3736-3746, September 2017.

[33]. W. Zhao, B. Zhou, M. Liu, W. Yao and T. Wang, "Decentralised Method for Solving Multi-area Stochastic Dynamic Economic Dispatch Problem," Journal of Engineering, vol. 2017, no. 13, pp. 2356-2362, November 2017.

[34]. J. Li, Q. Pan, P. Duan, H. Sang and K. Gao, "Solving Multi-Area Environmental/Economic Dispatch by Pareto-Based Chemical-Reaction Optimization Algorithm" IEEE/CAA Journal of Automata SINICA, vol. 6, no.5, pp. 1240-1250, September, 2019. 
[35]. J. Qin, Y. Wan, X. Yu and Y. Kang, “A Newton Method-Based Distributed Algorithm for Multi-Area Economic Dispatch” IEEE Transaction on Power Systems, vol. 35, no. 2, March 2020.

[36]. X. Li, H. Zhang and Z. Lu, "A Differential Evolution Algorithm Based on Multi-Population for Economic Dispatch Problems With Valve-Point Effects” IEEE Access, vol. 7, pp. 95585-95609, July 2019.

[37]. J. Yu, C. Kim, A. Wadood, T. Khurshaid and S. Rhee, "Jaya Algorithm With Self-Adaptive MultiPopulation and Levy Flights for Solving Economic Load Dispatch Problems" IEEE Access, vol. 7, pp. 2137221384, February 2019.

[38]. K. Alli, A. M. Jubril and L. O. Kehinde, "Development of a Semidefinite Programming Weighted Sum Based Approach for Solving Stochastic Multi-objective Economic Dispatch Problems Incorporating CHP Units," IAENG International Journal of Computer Science, IJCS, vol. 44, no. 4, pp. 1-12, November 2017.

[39]. K. S. Alli, A. M. Jubril, L. O. Kehinde "Solving Stochastic Multiobjective Economic Dispatch Problems Incorporating CHP and Wind Units" The Caribbean Academy of Sciences - 21st General Meeting and Conference, 2018

[40] A. M. Jubril, "Economic-emission dispatch problem: A nonlinear weight selection in weighted sum for convex multiobjective optimization," Facta Univ (NIS) Series Maths Inform, vol. 27, no. 3, pp. 357-372, 2013. [41]. Z. Jin, F. Li, X. Ma and S. M. Djouadi, "Semi-Definite Programming for Power Output Control in a Wind Energy Conversion System," IEEE Transactions on Sustainable Energy, pp. 1949-1959, 2014. 